Coulibaly, Bassidiki. - Du crime d'être noir : un milliard de noirs dans une prison identitaire

Préface de Louis Sala-Molins. Paris, Éditions Homnisphères (« Latitudes noires »), 2006, 221 p., bibl.

\title{
François Warin
}

\section{(2) OpenEdition}

\section{Journals}

Édition électronique

URL : http://journals.openedition.org/etudesafricaines/7513

DOI : 10.4000 /etudesafricaines. 7513

ISSN : 1777-5353

Éditeur

Éditions de l'EHESS

Édition imprimée

Date de publication : 31 mai 2007

Pagination : 411-417

ISBN : 978-2-7132-2139-2

ISSN : 0008-0055

Référence électronique

François Warin, « Coulibaly, Bassidiki. - Du crime d'être noir : un milliard de noirs dans une prison identitaire », Cahiers d'études africaines [En ligne], 186 | 2007, mis en ligne le 08 juin 2007, consulté le 22 septembre 2020. URL : http://journals.openedition.org/etudesafricaines/7513 ; DOI : https://doi.org/ 10.4000/etudesafricaines.7513

Ce document a été généré automatiquement le 22 septembre 2020

(C) Cahiers d'Études africaines 


\title{
Coulibaly, Bassidiki. - Du crime d'être noir : un milliard de noirs dans une prison identitaire
}

\author{
Préface de Louis Sala-Molins. Paris, Éditions Homnisphères (« Latitudes \\ noires »), 2006, 221 p., bibl.
}

\section{François Warin}

1 Voici enfin le livre attendu, écrit par un « Noir », par un «tout-autre » (p. 106) et qui paraît justement au moment où, avec la crise des banlieues, le refoulé, le vieux passé colonial de la France font si violemment retour.

2 Fini de parler pour le compte d'autrui. C'est un « Noir » cette fois-ci qui prend la parole pour s'adresser à nous, à notre arrogance satisfaite d'Occidentaux, mais aussi au milliard de "Noirs ", à l'immense foule de ces résignés qui ont perdu toute dignité et s'imaginent n'avoir d'autre choix que de "collaborer ». Car l'auteur n'y va pas par quatre chemins dans ses attaques et ses outrances! Gageons que ce brûlot qui se lit d'une traite, que ce petit livre pugnace au titre incendiaire va faire son chemin, si toutefois il ne fait pas d'abord un tabac.

3 L'auteur s'appelle Bassidiki Coulibaly, il fait partie du groupe des Siamou du Burkina Faso, et ce fils de forgeron d'Orodara peut attaquer sans retenue les "génocideurs ", zélateurs du Coran ou de la Bible, car il sait attiser le feu et porter le fer là où il faut. Il n'y a sans doute pas de rois négriers dans son ascendance, mais tout ce qu'il sait et tout ce qu'il est ne lui a pas été donné mais a été conquis de haute lutte. "Homme sans passé » comme le héros du film de Kaurismäki auquel il se réfère, il s'est toujours tenu debout et ne veut rien devoir à personne. Il n'attend de salut que de la part de ce qu'il appelle les «hommes de cœur» et, bien évidemment, comme le héros du film, de l'amour.

4 Cet ouvrage n'est pas un livre d'histoire, et les bons esprits ne manqueront probablement pas de se gausser des raccourcis cavaliers, des naïvetés, exagérations et contradictions dont le livre est émaillé. Notre homme ne fait en effet pas dans la 
dentelle, mais son livre, proféré dans un seul souffle, est un cri d'écorché qui va droit au but : malheur à ceux qui ne l'entendraient pas !

5 Avouons-le, il y a longtemps qu'une lecture ne nous avait pas mis aussi pleinement en empathie avec ces fils de Cham maudits et condamnés depuis toujours, n'en finissant pas de "payer pour le crime d'avoir choisi la mauvaise couleur, la couleur indélébile ", restés " prisonniers d'un cercle infernal ", acceptés à la rigueur mais "à condition de ne jamais oublier qu'ils sont noirs", souffrant de la plus terrible forme de l'exil qui soit : l'exclusion, depuis toujours, « de la famille humaine ».

Comment aussi ne pas le reconnaître? Un long travail de mémoire ou plutôt, pour éviter le caractère trop souvent passionnel et conflictuel des mémoires, une réécriture de l'histoire qui donnerait à chacun sa place reste chez nous bien souvent à faire. L'École, celle d'hier en tout cas, nous a donné bonne conscience en nous faisant étudier les textes édifiants des hommes des Lumières, Les nègres de Surinam par exemple, en oubliant de nous dire que Voltaire, Montesquieu, Locke et les autres émargeaient auprès des compagnies négrières transatlantiques de Bordeaux, de Nantes, de la Rochelle ou de Liverpool.

7 C'est pourtant sans aucune complaisance qu'on voudrait examiner ce texte. Le fait de passer aux ressortissants du Tiers Monde leurs éventuels travers serait sans doute le pire des mépris; celui qui d'égal à égal nous regarde en face et lance ses invectives le sait bien, et il aurait mauvaise grâce à ne pas nous laisser risquer quelques remarques :

8 1. La thèse du livre est claire et plusieurs fois martelée : la traite et la colonisation, profitant de la mansuétude et du sens de la parenté des Noirs, sont les causes de tous les maux et du sous-développement de l'Afrique actuelle (p. 162), et l'ont « déroutée de son évolution normale». On veut bien accepter cette thèse mais sous bénéfice d'inventaire.

9 2. Tous les peuples sans exception ont bâti leur identité en s'inventant un passé glorieux. Constituer l'identité d'une minorité sur la seule victimisation -comme si l'Afrique n'avait pas eu ses grandes heures, comme si l'identité noire n'était pas lumineusement, glorieusement présente dans la puissance d'exception de sa sculpture - est un pis-aller et une facilité qui peuvent devenir dangereux puisqu'elle réclame vengeance, exige et recherche des boucs émissaires. Non seulement le racisme cesse alors bien souvent d'être le monopole du groupe dominant, mais en attribuant tous ses malheurs à la traite et aux Blancs - aux " leucodermes »- on s'incline, comme le disait Césaire, devant la fatalité du sous-développement au lieu de se prendre en main, et on donne ainsi un merveilleux alibi à une classe politique corrompue qui fonctionne au népotisme et à la simonie.

10 Se faire passer pour descendant d'esclave et se faire appeler, comme le demandent certains, Indigènes de la République, ressemble à une plaisanterie qui essaie de donner indûment une crédibilité à l'équation suivante: discrimination $=$ colonisation $=$ esclavage. Car comment peut-on penser qu'au bout de cinq à six générations il soit possible de dire qui est descendant d'esclave, qui est descendant de maître ? De toute façon, à supposer que la notion de responsabilité collective puisse conserver un sens en traversant les siècles, on ne voit pas qui devrait demander pardon et à qui il le devrait (aux Antillais sans doute, pour les Africains c'est un autre problème...). Mutatis mutandis, s'il pouvait être établi chez nous qu'un tel est descendant de "Gaulois", 
serait-il fondé pour autant à demander des comptes aux descendants des "Francs " envahisseurs ou aux Romains impérialistes qui leur ont imposé leur langue?

11 3. Le contre-torpilleur, écrivait Montherlant, est toujours aussi nécessairement un torpilleur. Aussi on peut se demander si l'auteur, à son corps défendant peut-être, n'enferme pas à son tour l'Occident dans une prison identitaire qui ressemble à s'y méprendre à celle dans laquelle un milliard de "Noirs » ont été enfermés depuis des siècles. Ne réitère-t-il pas à l'égard de «l'Occident » cette opération qu'Edward Saïd avait justement dénoncée à propos de la fabrication de «l'Orient»? Rendant « coup pour coup", l'auteur semble nous dire avec la même bonne conscience: "Tu as fabriqué l'entité "Noirs", c'est à mon tour maintenant de fabriquer l'entité "Blancs" » et de remplacer votre histoire tout en rose par une histoire tout en noir ! Et réécrivant l'Histoire, il nous raconte comment, après les «Soudards verts » de l'islam, c'est la Férocité blanche des Européens qui "se sont invités à la curée ", "prenant d'assaut le continent ", le "saignant à blanc", se livrant à une "chasse à l'homme et à un pillage systématique », se jetant " avec avidité, cupidité, férocité » sur le nouvel eldorado qu'ils venaient de découvrir, tout cela se perpétrant aujourd'hui avec la complicité des potentats locaux corrompus mis en place par l'impérialisme. Et cela est vrai et ce n'est pas bien sûr l'Histoire (l'historiographie) que l'on apprend sur les bancs de l'École des Blancs car l'Histoire, dit-il, a été "écrite par les bourreaux » et continue d'être soigneusement falsifiée. Mais «L'Occident ", ce que l'auteur appelle ainsi, n’a jamais été " un ». S'il a été en effet le plus important foyer de domination, il a été aussi le lieu d'une contestation permanente de lui-même, le foyer par excellence de l'émancipation humaine et, dans la longue histoire des traites, il faut bien reconnaître que sa seule spécificité est d'avoir été, grâce à l'inflexibilité de Victor Schoelcher, abolitionniste ${ }^{1}$.

12 4. Mais Bassidiki Coulibaly est un justicier qui, sans trop de justesse et de justice, entend légiférer sur tous les crimes du passé. "L'Occident » a toujours « empesté la haine de l'autre» (p.134); alors pourquoi donc ne supporterait-il pas lui-même d'être haï ? demande-t-il. Ne faut-il pas rendre coup pour coup plutôt que de continuer à "supporter l'oppression"? On a toujours le sentiment que l'auteur, s'identifiant volontiers à Toussaint Louverture, à Franz Fanon ou aux Panthères noires se trompe d'époque et de combats. L'âge de la colonie n'est-il pas passé, et également celui de la néo-colonie? Il s'agit maintenant, avec la poussée des particularismes et l'arrivée en Europe de minorités aux mémoires de victimes, d'essayer, sous peine de voir se décomposer des États qui n'avaient pas vocation à devenir des terres de migration, de poser les fondements d'une convivence, comme on dit en haut-provençal, d'un vivre avec plus qu'un vivre ensemble, d'un partage des singularités et des différences susceptible de produire un Universel qui ne soit pas seulement blanc et européen mais qui soit enfin enrichi d'autres composantes. Mais l'Occident, nous dit-on, est narcissique, sa méconnaissance tragique de l'Autre le porte à ne respecter que l'Autre assimilé, que l'Autre devenu le Même, Achille Mbembe l'a lumineusement montré. "L'étranger (en somme) ou on le mange ou on le vomit. » Bassidiki Coulibaly ne veut manifestement pas être mangé, il ne veut pas s'identifier aux normes d'un modèle républicain déjà constitué et c'est lui qui à son tour semble nous vomir.

13 5. On ne reprochera pas à Sala-Molins, son mentor et son préfacier, l'immense générosité qu'il a toujours montrée à l'égard des «moins que rien» du Tiers Monde, mais à force de persuader les élites africaines qu'elles sont des victimes, à force de les pousser à se lamenter et à demander des dédommagements et des droits, ne les invite- 
t-on pas à se replier sur leur passé, à justifier au meilleur compte leur sousdéveloppement en les empêchant d'affronter les vrais problèmes du présent ? Et puis défendre les «Noirs » autorise-t-il pour autant à parler sur le ton d'une dérision au goût plus que douteux des juifs et de la Bible et à relancer implicitement la plus indécente des concurrences, la concurrence victimaire? «Les "Noirs" sont les seuls à avoir connu le pire" affirme également sans sourciller Bassidiki Coulibaly - en se gardant heureusement de tomber dans l'antisémitisme effectivement "nauséabond " d'un Dieudonné ${ }^{2}$ - comme si la traite lui appartenait à lui, le « Noir » et ne concernait pas tout le monde.

Mais il semble pourtant que la hargne et l'emportement passionnel le conduisent quelquefois à proférer des contre-vérités qui enlèvent au livre de sa crédibilité et risquent de déconsidérer son auteur. Le livre de Pétré-Grenouilleau, par exemple, et contrairement à ce qu'écrit l'auteur, est un vrai livre d'histoire, cet historien n'est pas un "saltimbanque " et un "manipulateur de chiffres" (p. 192), les raisons de son succès ne sont pas les mauvaises raisons du nationalisme. Faute de pouvoir poursuivre les criminels du passé, faudrait-il s'attaquer à un historien du présent, le déférer devant les tribunaux pour révisionnisme, le sommer finalement de se taire? Force est de constater ici que la loi Gayssot en punissant le négationnisme, a mis en place une dynamique fatale à laquelle il sera toujours difficile d'échapper: toutes les lois mémorielles risquent d'apparaître comme des lois liberticides, des entraves à la recherche, des formes d'intimidation qui jettent un soupçon sur la validité de la vérité officiellement retenue.

6. Quand on prend la responsabilité de parler, d'écrire, de publier, on respecte le principe d'identité. Ce principe, curieusement fustigé par le philosophe dans le texte et écorné en effet par l'incohérence de son attitude, implique également de donner un sens déterminé aux mots et aux syntagmes et de le conserver tout au long du discours : parler d'un "génocide utilitariste", comme Sala-Molins, c'est parler d'un "cercle carré ». Quand on exploite la force de travail d'un esclave, on ne peut viser sans contradiction son anéantissement. L'intérêt commande non de l'exterminer (génocide) mais de l'entretenir. C'est même parce que les Indiens du Brésil (après les serfs européens décimés par la malaria), trop fragiles, avaient succombé dans les champs de canne à sucre, que les Portugais les ont remplacés par une force de travail plus résistante. Le Brésil qui n'était pas une colonie de peuplement, s'est même constitué, si l'on en croit Gilberto Freire (Casa grande e senzala), à la cuisine, dans ce creuset, où le maître blanc et l'esclave noire se sont rencontrés : il n'y a pas de Brésilien aujourd'hui qui n'ait une goutte de sang noir. Gorée ou Ouidah sont des hauts lieux sur le chemin du martyrologe de l'humanité, des lieux témoins de la plus monstrueuse des institutions. Mais Gorée, lieu mythique de la traite esclavagiste au demeurant, n'est pas Auschwitz et le Code noir n'est pas la solution finale.

7. Ce livre frémit de bout en bout de cette impatience juvénile qui veut avoir tout, tout de suite et que soit réalisé sur le champ l'optimum éthique de la morale de conviction que $\mathrm{M}$. Weber opposait à la morale de responsabilité. Il faut rappeler pourtant que dans le champ de forces de la réalité, le combat pour la justice et la liberté passe par le conflit des intérêts et des passions et que c'est par étapes, à travers des compromis, que les choses finissent par changer. Mais à écrire l'histoire avec son cœur on risque de n'y rien comprendre et l'on va en effet d'indignation en indignation. Cet angélisme a toute notre sympathie, mais avec de bons sentiments, on risque de faire de la mauvaise 
historiographie. Projeter rétroactivement dans le bruit et la fureur du passé des notions juridiques, qui n'ont pris sens qu'avec nous qui les avons peu à peu élaborées et qui sont par définition inapplicables aux acteurs du passé qui ne sont plus, c'est tomber dans cet anachronisme qui, avec la pratique lénifiante de la repentance, est le tombeau de l'historiographie.

L'idéal universaliste de la République vient de connaître une crise sans précédent qui a fait trembler dans leurs fondements la totalité de ses institutions, à commencer par l'École. Le système républicain est aujourd'hui complètement bloqué et verrouillé par des élites qui l'ont confisqué à leur profit et qui ne cherchent qu'à s'auto-reproduire. Cinquante ans après la décolonisation, le pouvoir politique et médiatique n'accorde encore aucune "visibilité » aux minorités venues de son ex-empire colonial. La "République » restera une façade idéologique au nationalisme honteux tant qu'elle continuera ainsi à interdire de fait toute mobilité sociale.

Aussi est-il singulier de constater que, comme le remarquait Michel Wieviorka, ce sont aujourd'hui les "communautés" qui viennent au secours de la République et qui l'invitent à retrouver la souplesse et le dynamisme créateur dont on a fini par la priver. Les imams, dans les banlieues, cherchent à calmer les émeutes de la jeunesse, les organisations islamiques vont en Irak défendre le modèle français de laïcité, les plus hautes autorités de l'État défilent aux côtés des organisations juives et se rendent à la synagogue pour stigmatiser un crime particulièrement barbare. Que ce soit maintenant un « Noir » qui nous rappelle à l'ordre en retournant contre la République ses propres principes et en pointant toutes les omissions dont notre histoire s'est rendue coupable, relève de la même logique : en cherchant à mobiliser la communauté imaginaire des «Noirs» il ne s'oppose pas aux principes républicains, il conteste justement au contraire l'essentialisation de la différence ethnique et l'enfermement dont cette " communauté » pourrait être l'objet - par des regroupements défensifs ou des mesures de discrimination positive, par exemple - de telle sorte qu'à lire cet écrivain noir en butte à la ghettoïsation (enfermé dans une "prison identitaire»), on a envie quelquefois de s'écrier : mais «nous sommes tous des Noirs burkinabè ! ». C'est en effet paradoxalement au nom d'une identité particulière qu'il s'insurge contre ce qui pourrait ressembler à un communautarisme ${ }^{3}$ ou à une ségrégation, sans s'apercevoir cependant que c'est d'abord lui-même qu'il enferme dans une "couleur » et dans une communauté de ressentiment. Mais il reste qu'on ne peut qu'être édifié de voir ce ressortissant du Sud se sentir si bien chez lui dans une langue qu'il habite avec bonheur $^{4}$ et de le voir argumenter en jouant sur tout le clavier d'une culture universelle qui est aussi, au meilleur sens du mot, universitaire. L'ouvrage qu'il va publier sur Sartre, le plus cartésien de nos philosophes ${ }^{5}$, montre à quel point il a intériorisé cet individualisme frondeur et intransigeant qui, pour le meilleur et pour le pire, fait partie depuis si longtemps de l'idiosyncrasie nationale. «Philosophes, vous êtes de votre Occident! » écrivait Rimbaud.

19 Ce serait bien sûr avoir le triomphe indécent et le narcissisme bien primaire que de se satisfaire d'un tel constat et d'oublier les stigmates que porte ce livre: ceux d'une souffrance qui vient des entrailles, d'une souffrance accumulée tout au long de vingt ans de galère dans notre pays, ceux aussi d'une fierté sourcilleuse qu'on imagine avoir été sans cesse humiliée. Cette souffrance fait que ce livre est nécessaire, lui donne sa légitimité, mais lui confère aussi la couleur de la nuit. Et si c'est un destin et une tragédie que d'être né noir, on comprend que cet écorché vif soit porté à ressasser les 
épisodes d'une histoire révolue. Mais n'est-ce pas cette rumination qui l'empêche aussi, malheureusement, de faire des propositions et de se projeter vers l'avenir?

\section{NOTES}

1. Voir F. FEDERINI, L'abolition de l'esclavage de 1848. Une lecture de Victor Schoelcher, Paris, L'Harmattan, 1998.

2. Seuls la frustration et le ressentiment à l'égard de l'intégration très réussie des Juifs de France peut expliquer la dérive des extrémistes importée par Nation of Islam de Louis Farrakhan (ainsi au moment du meurtre particulièrement atroce d'Ilan Halimi la violence très typée de cette revendication : « S'il vous prenait l'envie d'effleurer ne serait-ce qu'un seul des cheveux du frère - Youssouf Fofana -, nous nous occuperons avec soin des papillotes de vos rabins »). Où est le temps où Frantz Fanon proclamait : «Quand un noir entend dire du mal d'un juif, il doit ouvrir les oreilles car c'est de lui qu'on parle. » « Depuis l'an 2000 quelque chose tourne vraiment mal entre les Juifs et les Noirs ", avoue Abdoulaye Barro, fondateur de l'association JUAF qui avait ouvert depuis longtemps un salutaire contre-feu à la bêtise et à l'intolérance. 3. Invention des Canadiens postérieure à la liquidation des Indiens, le communautarisme implique à proprement parler l'existence de communautés homogènes qui se ferment sur elles-mêmes en produisant leurs propres institutions. On imagine mal la République française, régressant à un état prénational, reconnaître à la communauté musulmane le droit d'appliquer la charia!

4. Que vive donc la francophonie ! La dénoncer comme néo-coloniale serait aussi intelligent que de dénoncer le jazz parce qu'il est héritier de la traite. Il faudrait cependant déconnecter le pays véritable qu'est toute langue de tout sol déterminé afin que la « francité » se libère de tout relent de nationalisme. On peut se sentir chez soi (sich heimisch fûlhen dit Heidegger qui développe cette idée) dans la langue sans avoir d'attachement ou de lien particulier avec un sol (Boden), ici avec le sol français, et c'est ainsi que le poète de l'exil, Saint John Perse, peut déclarer : La France... « est pour moi l'espèce sainte, et la seule, sous laquelle je puisse concevoir de communier avec rien d'essentiel en ce monde [...] la langue française (est) pour moi la seule patrie imaginable, l'asile et l'antre par excellence [...] le seul lieu "géométrique" où je puisse me tenir en ce monde pour y rien comprendre, y rien vouloir ou renoncer » (cité in HEIDEGGER, La dévastation et l'attente, Gallimard, 2006, p. 80).

5. Mais celui aussi avec qui, après la guerre, comme un repentir à son silence sous l'Occupation, l'esprit critique de l'Occident bascule dans la haine de soi et un masochisme moralisateur. 